\title{
DAZ1 Gene
}

National Cancer Institute

\section{Source}

National Cancer Institute. DAZ1 Gene. NCI Thesaurus. Code C124847.

This gene is involved in spermatogenesis. 